\title{
Research of Metro Guidance System Design Based on Visual Search Mechanism
}

\author{
Jiahui Wang ${ }^{1 *}$

\begin{abstract}
${ }^{1}$ School of Design Arts \& Media, Nanjing University of Science and Technology, Nanjing, Jiangsu 210094, China *Corresponding author. Email: $782605543 @ q q . c o m$
\end{abstract}

\begin{abstract}
In order to improve the present metro guidance system, which have no obvious information and reasonable spatial layout, the theory model of visual search is introduced into the research of metro guidance system design. By analyzing the visual search mechanism of human in the space of metro, the design principles of metro guide system are put forward to improve the efficiency of visual search. Improved design methods are put forward for the plane vision elements and space location elements of the metro guidance system, and the design system of the metro guidance system is constructed. It is of theoretical significance to the design of metro guidance system.
\end{abstract}

Keywords: metro guide, visual search, plane visual elements, spatial location

\section{INTRODUCTION}

Metro station is a place where people gather in high density. The metro guidance system can provide passengers with a variety of guidance information, and help passengers to locate in the underground space where there is no environmental reference, so as to realize a more reasonable direction of travel. However, there are some problems in the existing metro guidance system, such as the inconspicuous presentation of the plane information of the guide signs and the unreasonable location of the guide signs, which greatly reduce the visual search efficiency and affect the passengers' pathfinding efficiency.

The existing research of metro guidance system design mainly starts from the perspectives of interaction design, color design, ergonomics design, which plays a positive role in the design of metro guidance system. Cao xin (2014) applied the thinking and method of interactive design to put forward the design idea of constructing interactive digital multiplexing system [1]. Su yafei (2015) focused on the humanized design of the color information function planning in the metro guidance system by taking color elements as the entry point [2]. Xu zejun et al. (2018) proposed general design guidelines for space lighting and color design in guiding environment by analyzing the needs of the elderly in the process of pathfinding [3]. However, the existing researches seldom pay attention to the effectiveness of the spatial location layout of guiding signs, and at present, few scholars take the visual search mechanism of human beings as the starting point to design the metro guiding system on the basis of studying the visual search behavior and visual cognitive law of human beings.

Based on the theoretical model of background guidance, this study analyzes the visual search mechanism of people in the metro guidance environment. This paper puts forward two design principles of metro visual guide system which can improve the efficiency of visual search, and on this basis summarizes the design methods of plane visual elements and spatial location elements of metro visual guide system, and constructs the design system of metro visual guide system. It has a positive effect on creating a more reasonable metro guidance environment, reducing users' cognitive cost, and improving ride experience and route finding efficiency.

\section{ANALYSIS OF VISUAL SEARCH MECHANISM IN METRO SPACE}

Visual Search refers to the process in which an individual actively searches for a target object from a complex visual environment. It is a visual behavioral process of a specific stimulus and a complex cognitive process [4]. Human beings are engaged in visual search activities every day, and any small reduction in search time may result in huge cost savings and effective improvement of user experience [5]. By studying human visual search mechanism, we can better understand how humans perceive, search and process visual information, and provide effective suggestions for designers of artificial visual search tasks [6].

The contextual guidance model of visual search explains the relationship between real scene and stimulus recognition, that is, when people conduct visual search in real scene, they will receive the dual functions from two aspects, one is background information, the other is knowledge and experience [7]. The recognition of the scene and the target by the observer is realized by the integration of two parallel processing pathways: local processing pathway (bottom up) and global processing pathway (top down). Local feature processing is based on background information such as color and size. The extraction of these local features forms a visual dominant region and affects the information search of the observer. 
The whole feature processing is based on the whole feature extraction, activates the existing knowledge and experience, and adjusts the direction of information search. In this way, bottom-up and top-down processing is used to fit and ultimately determine what information the observer searches for [8] (Figure 1).

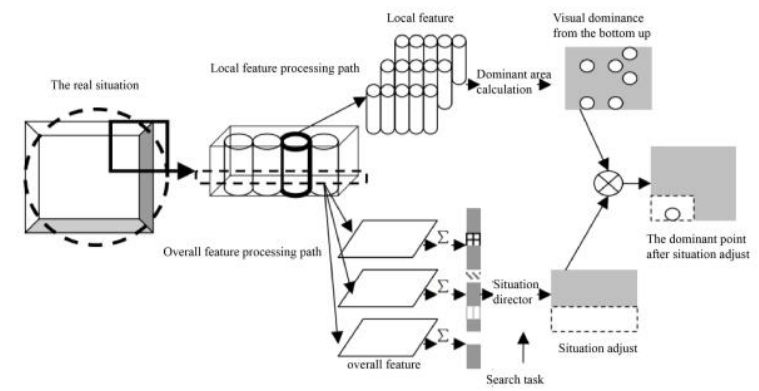

Figure 1 A theoretical model of background guidance for visual search

According to this model, when people search for visual information in the metro environment, on the one hand, they will be stimulated by the visual information with emergent dominance in the scene to form a visual dominant area and attract people's attention. On the other hand, according to the task target and their own experience and knowledge, they will actively search and pay attention to the area of interest, form the prediction of spatial position, and generate the expected clues of "target stimulus in a certain position", which will play a regulating role on the scanning path, and finally determine the direction of visual search [7].

\section{DESIGN METHODS OF METRO GUIDANCE SYSTEM BASED ON VISUAL SEARCH MECHANISM}

\subsection{The Design Principle of Metro Guidance System}

Based on the visual search mechanism of people in the metro visual environment, this study proposes two design principles to be followed in the design of the metro visual system.

\subsubsection{The Flat visual elements should have visual significance}

For the local feature processing process of visual search, the graphic design of metro guide sign is required to have visual significance to promote the search speed of local feature processing path. When people focus on the scene, visually prominent objects, such as size, color, brightness, texture, contrast and movement direction, have the advantage of visual competition and can obtain earlier gaze [9]. Therefore, in the design of metro guide signs, it is necessary to enlarge the guide information appropriately, use bright colors, obvious contrast, enhance the sense of movement and other ways to highlight important information, so that important information in the visual search of local feature processing process to form a visual advantage area, the first to attract people's attention.

\subsubsection{The spatial elements should conform to people's psychological expectations}

For the overall feature processing path of visual search, the location layout of metro visual information needs to fit the direction prediction formed by people based on previous knowledge and experience, that is, the design of metro visual system needs to match the common sense and visual habits of users. On the one hand, it conforms to people's general cognition of the spatial layout of metro guide signs, on the other hand, it conforms to people's visual search habit in the space. In order to promote the search speed of the whole feature processing pathway, the corresponding guiding information is set in the expected position of human psychology, so that human can accurately find the target information in the expected position.

\subsection{Construction of Metro Guidance System Design}

The design of the metro guidance system is mainly reflected by color, graphics, text, lighting and other plane visual elements and spatial location elements. Design the elements properly to disseminate the correct guidance in the environment. Based on the visual search mechanism of people in the metro guiding environment, under the guidance of the above two design principles, specific design methods to improve the visual search efficiency are proposed for each design element of the metro guiding system, and the design system of the metro guiding system is constructed (Figure 2).

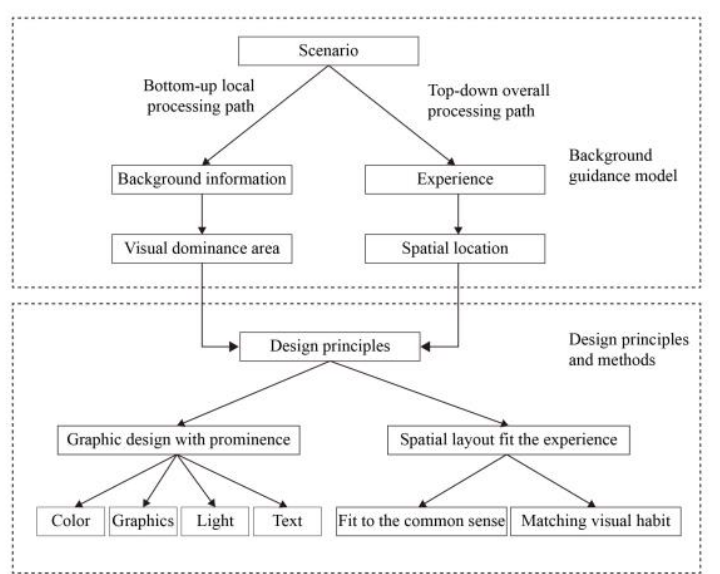

Figure 2 Construction of design system of subway guidance system 


\subsubsection{Graphic Visual Element Design Method with Visual Significance}

(1) Highlight important information with prominent colors to attract users' attention. Color is the most sensitive visual information element and the key factor for rapid visual focus. Different colors are perceived differently by visual perception. In the design of the guiding system, the color is too uniform, which will make the whole guiding system sink into the whole environment, thus failing to meet the requirements of remote viewing, thus losing its effective "indicating" function [10]. Therefore, in the design of guide signs, it is usually necessary to use color to highlight the strong stimulation of information, such as on the white wall supplemented by red graphics, through contrast to generate striking feelings, attract people's attention, in order to improve the visual search efficiency.

(2) Enhance the guiding function of the logo with the visual tension of graphics. Graphics are generally a concise symbol to express a certain meaning. Compared with text, the recognition of graphics is more intuitive and vivid and easy to understand. Therefore, instead of text barriers, specific information can be transmitted quickly and accurately [11]. Enhancing the visual tension of the graphics helps to enhance the guiding function of the logo, and makes it easier for people to focus concise graphics from the visual center. During the design of the guidance system, the visual tension of the graphics can be enhanced by making the motion of the graphics and magnifying them [12]. In addition, the three-dimensional logo beyond the plane, with the help of three-dimensional modeling to strengthen people's association of the space environment also helps to attract visual attention and improve visual search efficiency.

(3) Reduce users' cognitive cost with bold and enlarged isometric font. In the complex underground environment, noisy auditory background, time pressure and other factors will increase the difficulty of text recognition. In this situation, the design of text information is required to be easy to identify quickly, and the text is required to be visually significant in the design of font, size and color. The font should be as plain as possible, and the text can be highlighted by bolding and magnifying. In contrast to make the guide sign has the aesthetics and USES the small font size, should adopt the reasonable amplification way to make it easy to identify.

(4) The lighting design is mainly based on soft indirect lighting and focuses on important information. The lighting of metro space should be mainly indirect lighting and supplemented by direct lighting. This kind of light is relatively soft and comfortable for human eyes, which is conducive to creating a warm and comfortable humanized space, and will not cause strong reflection and affect the identification of identification information. In addition, should undertake key illume in important information place, wait for special target object with emphasis exit, stair, guide sign, achieve the action of visual guidance thereby.

\subsubsection{The Spatial Layout Design Method That Conforms to The User's Psychological Expectation}

The expected direction of people's visual search in the metro guiding space is influenced by people's general cognition of the spatial layout of the metro guiding system on the one hand, and by the subtle visual search habit on the other hand. The spatial layout design of metro guide signs should take these two aspects into consideration.

(1) The design of the metro visual guide system should match with people's cognition of the spatial layout of the metro visual guide system.

In the metro guiding environment, different spatial positions correspond to different forms of signs, which have their own common uses and fixed display contents. The interior space elements of the metro include walls, pillars, ceiling and floor, so according to the fixed position of signs, they can be divided into wall signs, signs on pillars, hanging signs, landmarks and standing signs [13]. The pointing signs on the wall are generally placed with the guidance information and map information of each exit; The signage on the column usually sets the transfer guide information, the guide information of the toilet and the elevator, and the name of the station. The suspended signboards are always placed on the metro transfer stairs and at the corner of the passage, and the information of inbound, outbound and transfer directions with arrows is usually placed. Most of the landmarks are the pointing arrows of the transfer routes. Standing signs are placed on fixed shelves on the ground, usually with maps of metro lines with the names of each station and a map of the area. Different spatial positions correspond to different forms of signs and the conventional setting contents of all kinds of signs, and people have formed a basic cognition and judgment on this. Therefore, when people want to search the expected target information, they will go to a specific spatial location to look for a specific form of signage. For example, if they want to search for inbound and outbound information, they will generally pay attention to hanging signs, and if they want to search for a large area of map information, they will usually look for a standing sign. According to different visual search task, the different space position distribution to the obvious differences in visual attention, and identification of space position and represents a specific type of guidance information, so you cannot blindly change identity information types corresponding relation with the space location and identity, otherwise it will conflict with people experience of common sense, greatly reduce the visual search efficiency. For example, if people think that advertising information will appear on the hanging tag, don't blindly ground identification as the main source of information, so can't through the ground on the attached type identification information instead of suspension's identity information, only as a supplement and to emphasize information auxiliary way, people won't be able to timely according to the psychological expectations location to find specific identity. 
[3] Xu Z.J. Cognitive research on subway guidance environment under the background of aging $[\mathrm{J}]$. Decoration, 2018 (09) : 82-84.

[4] Sun Q. Interaction between scene context and target template in visual search [J]. Psychological science, 2014 (2) : 265-271.

[5] Gong Y. Effect of color composition on visual search efficiency of graphic symbols [J]. Journal of computer-aided design and graphics, 2016, (07) : 11151120 .

[6] Liang J. History, current situation and future of visual search theory [J]. Social psychological science, 2004 (3) : 3-8.

[7] Bai X.J. Theoretical model and research review of stimulus recognition in real situations [J]. Advances in psychological science, 2008,16 (5) : 679-686.

[8] Wang H.Y. Act-r cognitive model analysis of icon visual search behavior [J]. Journal of computer-aided design and graphics, 2016,28 (10) : 1740-1749.

[9] Shi H.Q. Effects of visual significance and reward value on sacculturation [J]. Psychological science, 2016 (4) : 862-868.

[10] Zhou Y. Research on the application of color in subway visual guide system [J]. Art and technology, 2014 (6) : 230-230.

[11] Chen S.C. Research on urban vision-oriented design -- a case study of changsha metro [J]. Chinese \& foreign architecture, 2012 (9) : 37-40.

[12] Gong Y. Effect of color composition on visual search efficiency of graphic symbols [J]. Journal of computer-aided design and graphics, 2016,28 (7) : 1115-1120.

[13] Bao N. Investigation and analysis of Beijing metro station signage system [J]. Urban rapid rail transit, 2009,22 (6) : 23-28.

\section{REFERENCES}

[1] Cao X. Interactive design considerations in the design of Beijing metro guidance system [J]. Packaging engineering, 2014 (6) : 37-40.

[2] Su Y. An analysis of the humanization of the color information function in the subway guidance system [J]. Industrial design, 2015 (10) : 109-110. 